\title{
Results of Tests of Three-Dimensionally Integrated Chips Bonded to Sensors
}

\author{
Grzegorz W. Deptuch, Senior Member, IEEE, Gabriella Carini, Terence Collier, Paweł Gryboś, Member, IEEE, Piotr \\ Kmon, Member, IEEE, Ronald Lipton, Piotr Maj, Member, IEEE, Marcel Trimpl, Member, IEEE, David P. Siddons, \\ Robert Szczygieł, Member, IEEE, Raymond Yarema, Life Member, IEEE,
}

\begin{abstract}
The VIPIC1 pixel readout integrated circuit was designed for the requiring high timing resolution, pixel based, $\mathrm{X}$ ray Photon Correlation Spectroscopy experiments, that are typically conveyed using $8 \mathrm{keV} \mathrm{X}$-rays at a synchrotron radiation facility. Technical hurdles related to a very tight wafer bonding alignment requirement resulted in delivery delays of 3D bonded wafers. Recent improvements in alignment of wafers bonded with the $\mathrm{Cu}-\mathrm{Cu}$ thermo-compression method and the $\mathrm{Cu}-\mathrm{DBI}{ }^{\circledR}$ bonding method resulted in operational devices that have been tested. These tests include chips bonded to silicon pixelated sensors and tested with X-ray sources, for the first time. Results of these tests are presented.
\end{abstract}

\section{INTRODUCTION}

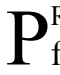
ROTOTYPES of three-dimensionally integrated circuits for a few selected applications were submitted for fabrication on the 3D multi-project wafer (MPW) run that was managed by Fermilab [1]. The Vertically Integrated Photon Imaging Chip (VIPIC) project aims at providing a high timing resolution, pixel based readout for X-ray Photon Correlation Spectroscopy experiments [2] at a synchrotron radiation facility. VIPIC1 is the first integrated circuit that was designed and fabricated for the purpose of detection of $8 \mathrm{keV}$ X-rays, operating without dead time and providing hit positions and

Manuscript received June 22, 2014; revised XX X, 2014

Fermilab is operated by Fermi Research Alliance, LLC under contract No. DE-AC02-07CH11359 with the U.S. Department of Energy. BNL is supported by the U.S. Department of Energy, Office of Science, Office of Basic Energy Sciences, under Contract No. DE-AC02-98CH10886. AGHUST was supported by National Science Center, under Contract DEC2011/01/B/ST7/05155 and by EU FP7 AIDA program (Grant Agreement Number 262025) and Polish Ministry of Science and Higher Education (Project number 2225/7.PR/2011/2).

G. Deptuch, R. Lipton, M. Trimpl and R. Yarema are with the ASIC Development Group of the Electrical Engineering Department of the Particle Physics Division at the Fermi National Accelerator Laboratory, BP 500, Batavia, IL 60510, USA, (telephone: +1 630840 4659, fax: +1 630840 2950, e-mail: deptuch@ieee.org, lipton@fnal.gov, trimpl@fnal.gov, yarema@fnal.gov).

D. P. Siddons is with the Photon Sciences Directorate at the Brookhaven National Laboratory, BP 5000, Upton, NY 11973, USA, (e-mail: siddons@bnl.gov).

G. Carini is with the SLAC National Accelerator Laboratory, 2575 Sand Hill Road, Menlo Park, CA 94025, USA, (email: carini@SLAC.stanford.edu).

P. Gryboś, P. Kmon, P. Maj and R. Szczygieł are with the Department of Measurement and Electronics at the Faculty of Electrical Engineering, Automatics, Computer Science and Biomedical Engineering at the AGH University of Science and Technology, al. A. Mickiewicza 30, 30-059 Kraków, Poland, (pawel.grybos@agh.edu.pl, kmon@agh.edu.pl, piotr.maj@agh.edu.pl, robert.szczygiel@agh.edu.pl).

T.Collier is with CVInc, 850 S. Greenville, Suite 108, Richardson, Texas 75081, USA (tqcollier@covinc.com). their times with precision better than $10 \mu \mathrm{s}$.

The 3D MPW project suffered from significant delivery delays of 3D bonded wafers from fabrication. Recently, technical hurdles related to a need for exacting alignment of wafers during the bonding process were overcome. Improvements in alignment of wafers bonded with the $\mathrm{Cu}-\mathrm{Cu}$ thermo-compression $(\mathrm{Cu}-\mathrm{Cu}$ TC) method [3] as well as the $\mathrm{Cu}$ Direct Bond Interconnect $\left(\mathrm{Cu}-\mathrm{DBI}^{\circledR}\right)$ bonding method [4] yielded operational devices that were successfully tested and the next steps were thereby allowed. Delivery of this lot of wafers allowed progression to bonding of the pixel readout chips to the sensors. A few VIPIC1 chips were bonded to small size "baby' sensors, using an optimized solder bump bonding technology that was performed on individual readout and sensors dies. Pitches of $80 \mu \mathrm{m}$ and $100 \mu \mathrm{m}$, on the VIPIC chip and on the available sensors respectively, required deposition of a pitch adapting pattern of pads. This was properly taken care earlier when 3D wafers were undergoing steps of back-thinning and deposition of the back-side metal. By doing so, first results of tests with X-ray photons could be achieved earlier and are presented in the following paper. Currently efforts are underway to bond read out chips to 80 $\mu \mathrm{m}$ pitch sensors using the Ni-based chip-to-wafer $\mathrm{DBI}^{\circledR}$ technology.

The paper is organized in 5 sections. The first one is introduction. The second section provides a short description of the architecture and functionality of the VIPIC1 chip and discusses variants of bonding methods used for building the 3D chips. The third section reveals details of the bump bonding process that was used to mate a few VIPIC1 chips to small pixelated Silicon pixel sensors, featuring the $100 \mu \mathrm{m}$ pitch. The fourth section discusses results of tests of the chips that were performed using laboratory ${ }^{55} \mathrm{Fe}$ and ${ }^{109} \mathrm{Cd}$ radioactive sources, emitting soft $\mathrm{X}$-ray photons. The last section of the paper concludes and summarizes the presented material.

\section{ARCHITECTURE, FUNCTIONALITY AND METHODS OF BONDING USED FOR VIPIC1 CHIPS}

The topology of the chip meets the basic needs of experiments with synchrotron radiation beams. It also opens an evolutionary path of further developments. For example equipping every pixel with a measurement unit for accurate timing of an event with respect to the time frame of a much longer duration is foreseen as one of the next steps. At the present time, VIPIC1 registers radiation hits and assigns their occurrences to the coarse time frames that are defined by a 
slow clock delivered from the outside of the chip.

The VIPIC1 chip is a small prototype, the size of which resulted from the division of the reticle into $5.5 \times 6.3 \mathrm{~mm}^{2}$ blocks in the first 3D HEP MPW run. The chip features a matrix of $64 \times 64$ pixels, which is divided into 16 groups of 256 pixels (4 rows of 64 pixels each). A sparsification engine is implemented in every group. The sparsifier feeds the results to a serializer and data is output through LVDS drivers in parallel from each group. The pixel size is $80 \times 80 \mu \mathrm{m}^{2}$. The details of the chip architecture and further description of the operation of the chip were already provided elsewhere [5][6].

The first working chips were obtained from two pairs of wafers: one bonded with the $\mathrm{Cu}-\mathrm{Cu} \mathrm{TC}$ and one bonded with the $\mathrm{Cu} \mathrm{DBI}{ }^{\circledR}$ method. Both were delivered in June 2012. Using chips from this lot, the full functionality of the VIPIC1 chip could essentially be accessed. These results of tests, carried out on the circuits prior to connecting them to pixel sensors, were subjects of past communications [6][7]. Similarly, the test environment that was prepared using the NI PXIe system and the LabView language was also largely described [7].

Illustrations of the $\mathrm{Cu}-\mathrm{Cu} \mathrm{TC}$ and $\mathrm{Cu} \mathrm{DBI}^{\circledR}$ bonding methods with the dimensions of the elements making up the bonding interface, such as were used in the MPW project, are shown in Fig. 1. The difference between these two methods is the material that holds tiers together when they fuse.
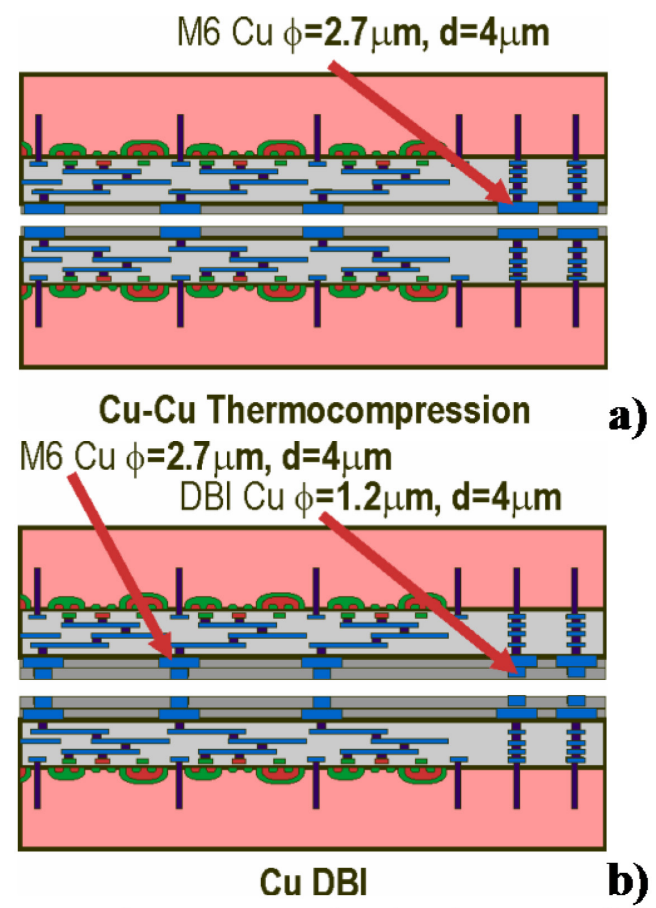

Fig. 1: $\mathrm{Cu}-\mathrm{Cu}$ themo-compression a) and $\mathrm{Cu} \mathrm{DBI}(\mathrm{B})$ bonding methods used on 3D chips.

In the $\mathrm{Cu}-\mathrm{Cu} \mathrm{TC}$ method, mechanical connection of two wafers is provided by $2.7 \mu \mathrm{m}$ in diameter $\mathrm{Cu}$ insets from the last metal of the process that are fused together [8]. In the $\mathrm{Cu}$ $\mathrm{DBI}^{\circledR}$ method, two wafers are held together by the fused oxide layers and electrical connections between tiers are obtained through smaller $1.2 \mu \mathrm{m}$ of diameter $\mathrm{Cu}$ plugs that are pressed one to another from both tiers. The bonding oxide and contact $\mathrm{Cu}$ plugs are added in the post-processing steps with extra masks required. The advantage of the $\mathrm{Cu} \mathrm{DBI}^{\circledR}$ method with respect to the $\mathrm{Cu}-\mathrm{Cu} \mathrm{TC}$ method is that the $\mathrm{DBI}^{\circledR}$ process does not require strong pressing of one wafer against another, nor elevated temperatures. It can be still reworked shortly after initial contact of wafers is established to correct for misalignment if such is detected. Additionally, it is more precise since the wafers show some convexing prior to bonding, which facilitates spreading of the bond from the center of wafers towards their edges.

In tests of the VIPIC1 chips, it could be concluded that better yields were observed on the chips bonded with the $\mathrm{Cu}$ $\mathrm{DBI}^{\circledR}$ method. However, the $\mathrm{Cu}-\mathrm{Cu} \mathrm{TC}$ method possesses some advantages, e.g. lower costs due to fewer operations required. Thus, it can be used in future as well.

The new batches of 3D wafers were delivered in March, May and June 2013. The last were 3D wafers were bonded using the $\mathrm{Cu} \mathrm{DBI}^{\circledR}$ method. VIPIC1 chips diced out from one of these last wafers were picked up for attachment of dies to sensors using the $\mathrm{Sn}-\mathrm{Pb}$ bump bonding method presented in this paper.

\section{BUMP BONDING PROCESS USED TO MATE A FEW VIPIC1 CHIPS TO SMALL PIXELATED SILICON PIXEL SENSORS}

The final goal for the project is to bond VIPIC chips to $80 \mu \mathrm{m}$ pitch pixel sensors that were built at the Brookhaven National Laboratory (BNL), to allow connection of all readout channels to sources of signals. And, later, to open back-side pads for bump bonding of sensor-readout assemblies onto a precise printed circuit board (PCB) [1]. These VIPIC sensors fabricated at the BNL are available on a wafer together with the designs for other $3 \mathrm{D}$ projects [1] and bonding of readout chips to sensors is planned using the die-to-wafer $\mathrm{DBI}^{\circledR}$ bonding process with Ni bond posts. However, it was decided to use small size "baby" sensors with $32 \times 38$ pixels laid out with the pitch of $100 \mu \mathrm{m}$ first in order to accelerate the tests of the VIPIC1 chip stimulated by X-ray photons. The "baby" sensors were available as single dies only. Their size was such that wire bonding to the pads located on the periphery of the VIPIC1 chip could still be achieved after their attachment to the chips. This was a significant simplification as most of the setup from the tests of the VIPIC1 chips could be reused [6]. In order to allow attachment of "baby" sensors, the $80 \mu \mathrm{m}$ pitch pads for the BNL sensors were overlaid with the new $100 \mu \mathrm{m}$ pitch pads. Thus the geometrical matching of both dies was achieved. Nevertheless, every fifth original pad had to be skipped in this procedure. The result is shown in Fig. 2, where a part of the layout of pads on the surface of the VIPIC1 chip can be seen. The big pads, having a diameter of $60 \mu \mathrm{m}$ and thickness of about $700 \mathrm{~nm}$ are to be connected to the pixels, while small pads are the skipped ones. The pads on the "baby" sensors had round opening of $60 \mu \mathrm{m}$, but their thickness was not exceeding $400 \mathrm{~nm}$. Pads on both components were made of Aluminum.

A solderable layer, called under-bump metallization (UBM), was added first to the aluminum pads independently on both the VIPIC1 and sensors dies. The process used consisted in adding a $\mathrm{Ni}-\mathrm{Au}$ layer on top of a very thin $\mathrm{Zn}$ layer that was built up first on Al. The thickness of the Ni layer was in the 
range from 2 to $4 \mu \mathrm{m}$ and an oxidization prevention layer of $\mathrm{Au}$, having thickness in the range from 0.05 to $0.15 \mu \mathrm{m}$, was deposited on top of it. The original small thickness of the $\mathrm{Al}$ pads resulted in certain difficulties in obtaining adequate adhesion of the $\mathrm{NiAu}+$ solder bump composition. A minimum thickness of the $\mathrm{Al}$ pad is required for robustness of the UBM deposition. If that layer is not thick enough, the catalyst in the zincation process will etch away too much of the Al layer preventing good adhesion. Each bump can exceed from 50 to 75 grams of shear force and if the adhesion is too weak a die might be destroyed.

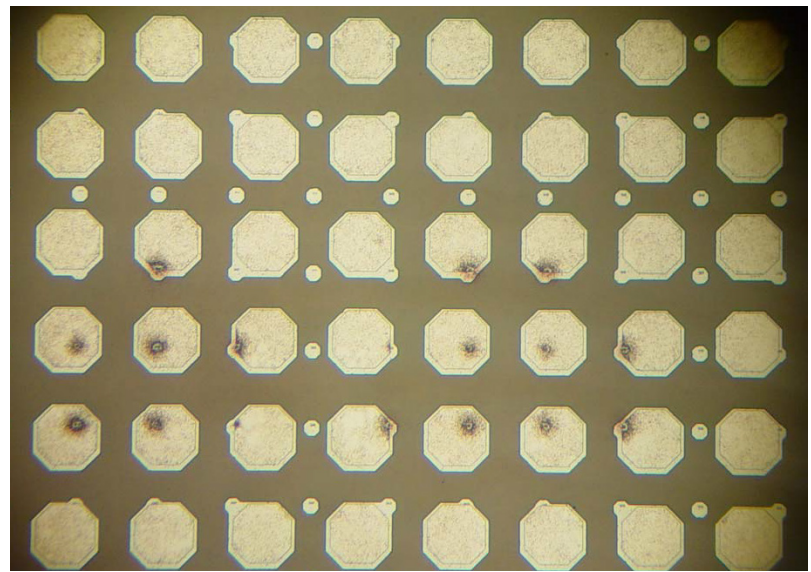

Fig. 2. Layout of pads on the surface of the VIPIC1 chip.

In our work, it was decided to deposit $\mathrm{Sn}-\mathrm{Pb}$ bumps on the sensor dies. The first few dies did not yield either good UBM or bumps with good adhesion. It was likely that the poor result was due to in-situ $\mathrm{Al}$ on the pads being thinner than $0.5 \mu \mathrm{m}$ as the metal was completely removed during the baseline process. First modification of the Ni-Au deposition process did improve the yield to over $85 \%$ of the pads retaining UBM and bumps. Additional modifications yielded over $95 \%$ of the pads and final processing yielded all pads with exception of the four peripheral pads that were destined for biasing of the detector. At the end, further modification yielded $100 \%$ bump of the pads, which can be seen in Fig 3. The bumps are eutectic $\mathrm{Sn}-\mathrm{Pb}$ with a height of $75 \mu \mathrm{m}$. It was not clear, why certain pads demonstrated more resistance to retain UBM but it was concluded that some electrochemistry could play a role there. As it can be seen in Fig. 4, where a VIPIC1 chip with a flip-chip bonded "baby" pixel sensor is shown, some bonding pads are darker. It was found that pads for ground connections and power supplies, were those on which UBM deposition did not work well.

The "baby" sensors were flip-chip bonded on the readout dies. The post reflow gaps were measured in the range from 45 to $50 \mu \mathrm{m}$. An organic epoxy underfill was added in the post flip-chip bonding operation due to the uncertainty of the UBM adhesion for additional mechanical strength of the assemblies. Generally speaking, underfill is critical for larger dies, made for example of dissimilar materials, that are to be attached together. There, coefficient of thermal expansions (CTE) could cause enough stress to result in failure at the solder joints. In our case it was used more as a precaution. The built assemblies were mounted on PCBs and wire bonded. Pads that were covered with $\mathrm{NiAu}$ layer were easily bondable.
Significant difficulties were experienced with adhesion of $\mathrm{Al}$ wires to pads with poor plating.

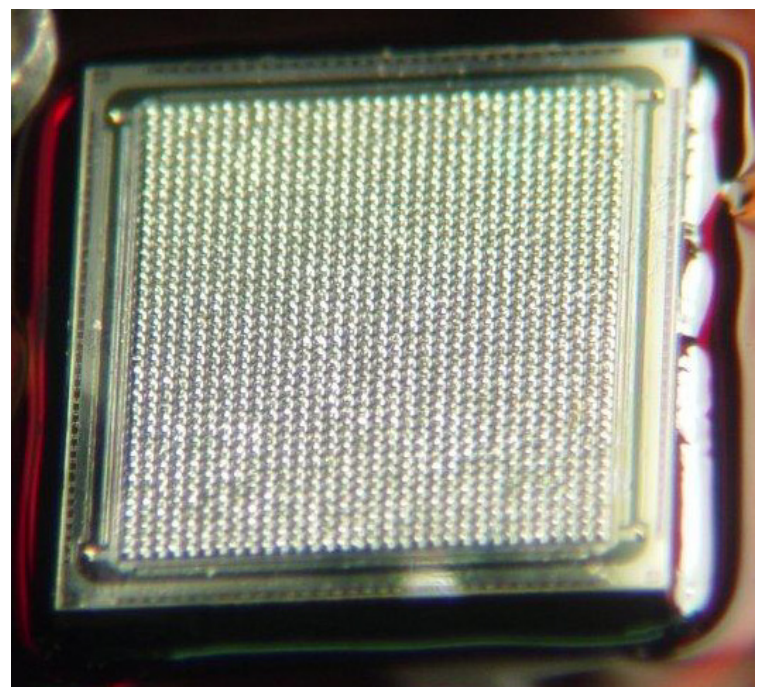

Fig. 3. Surface of the "baby" pixel sensors with $\mathrm{Sn}-\mathrm{Pb}$ deposited.

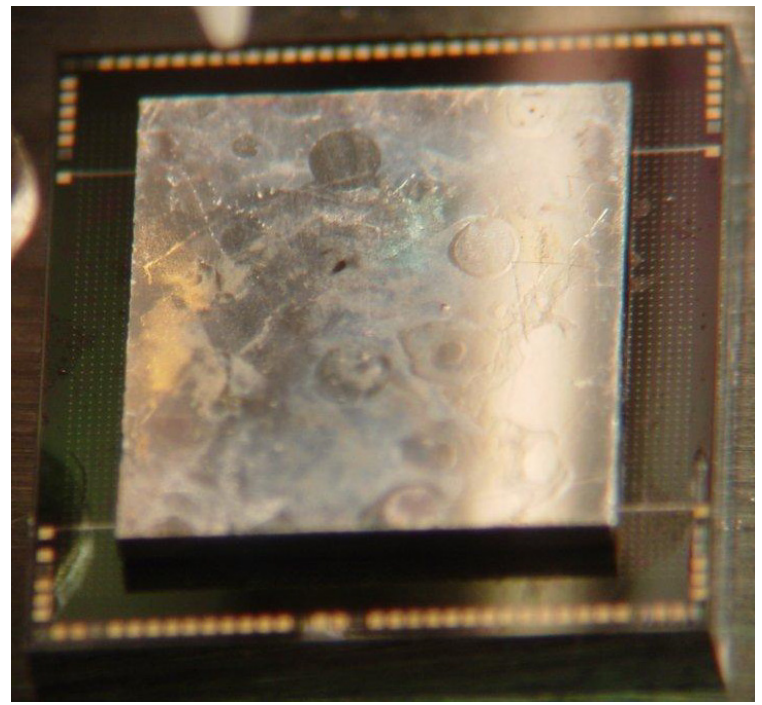

Fig. 4. VIPIC1 with flip-chip bonded "baby" pixel sensor.

\section{TESTS OF THE VIPIC1 CHIP WITH X-RAYS}

Testing of the VIPIC1 chips was performed using the NI PXIe system with FlexRIO cards driving $100 \mathrm{MHz}$ digital I/O modules. Power supplies and biases were also provided by the PXIe system with power supply and Digital-to-Analog Conversion (DAC) modules. The sensors were biased by applying $120 \mathrm{~V}$ and $210 \mathrm{~V}$ of the reverse bias voltage on their backplanes. The full depletion was achieved and leakage current (monitored by Keithley 2400 SMU) was in the range from a few tens of nanoamperes to about $200 \mathrm{nA}$ depending on the chip tested. Two radioactive sources, i.e. ${ }^{109} \mathrm{Cd}(1 \mathrm{mCi})$, emitting $22 \mathrm{keV}$ photons and ${ }^{55} \mathrm{Fe}(10 \mathrm{mCi}) \mathrm{keV}$, emitting $5.9 \mathrm{keV}$, were used in the tests. The data acquisition software was written in such a way that the chips could be tested in the full sparsified readout mode with simultaneous acquisition of the data from all 16 outputs. The data serialization clock was $50 \mathrm{MHz}$ and durations of time frames were varied from 1 to $80 \mu \mathrm{s}$. The hits were output normally at any duration of the 
time frame. The total number of hits varied from 3 to 250 from the shortest and the longest duration of the time frame tested, respectively. It could be concluded from observations of physical properties of the VIPIC1 output signals that faster serialization clocks could be used; however $50 \mathrm{MHz}$ was working well with the NI CMOS DIO modules in the range of easily adjustable signal latching delays. The VIPIC1 chips were tested at the nominal or close to nominal bias and power supply conditions.

\section{A. Transmission radiograms}

Acquisition of transmission radiograms was the first simple test that was performed. A small $\mathrm{W}$ mask with external dimensions of $2.5 \times 2.5 \mathrm{~mm}^{2}$, thus covering a group of $25 \times 25$ pixels, was used. The mask had a uniform thickness of $1 \mathrm{~mm}$, but it also had some features cut out in its center. Because of their smaller sizes than the pixel pitch on the chip and on the sensor bonded to it, the shapes details were expected to merge and to result in areas equivalent to intermediate exposure levels. First, a reference run without source was taken, and no hits were registered, except when thresholds were set at the noise levels. Then, a ${ }^{55} \mathrm{Fe}$ source was place atop of the mask and acquisition was run for a few hours with the same threshold settings. The run was long to accumulate enough of hit statistics. An example of the obtained results is shown in Fig. 5. The thresholds were set just above the noise level. Finding the required setting was relatively easy as a part of the VIPIC1 chip had no connections to sensor diodes, allowing to see at which threshold levels no entries could be detected in these parts. All summed counts, registered above the set thresholds, are shown in the image. The visible black lines are due to lacking signals from rows and columns that were not connected to the sensor diodes. The brightest pixels are holding up $2 \times 10^{9}$ hits registered. Graded intensities registered in the center of the mask correspond to the area with features carved in the mask.

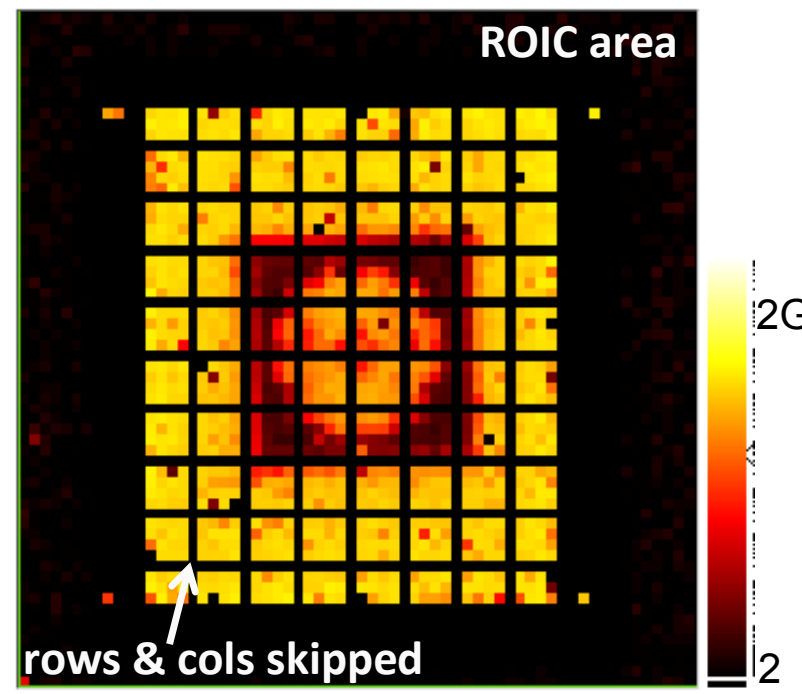

Fig. 5. Transmission radiogram of a small $\mathrm{W}$ mask $\left(2.5 \times 2.5 \mathrm{~mm}^{2}\right)$ placed atop of the sensor back-side illuminated and fully depleted.

Darker pixels in the fully exposed area are due to the presence of gain dispersions that was quantitatively studied further. It can be seen that only a small number of pixels are completely insensitive to the X-ray photons.

\section{B. Tuning of feedback resistance}

Estimation of quantities, like gain and noise with their pixel-to-pixel spreads, followed simple, conducted without optimization of the bias settings, acquisitions of the transmission radiograms. The tests aimed also at checking whether a system, built using the $3 \mathrm{D}$ technology, performs differently from the structures that are built using classic technologies. The nominal setting of externally controlled currents $\mathrm{I}_{\mathrm{CSA}}=32 \mu \mathrm{A}, \mathrm{I}_{\mathrm{SHA}}=14 \mu \mathrm{A}$ and $\mathrm{I}_{\mathrm{DIS}}=26 \mu \mathrm{A}$, resulting, after rescaling in current mirrors, in currents of about $4.65 \mu \mathrm{A}$, $0.35 \mu \mathrm{A}$ and $1.25 \mu \mathrm{A}$ in the first transistor of the preamplifier, of the tail current of the differential pair of the shaper and of the tail current of the comparator, were used first. When performing detailed, with $0.5 \mathrm{mV}$ precision, scans of threshold values applied to the pixel discriminators, it was observed that counts were also registered on the negative side of the noise peaks during exposures to the radioactive sources. Examples of the registered parts of the integral spectra, showing signals on the negative side of the noise peaks, are presented in Fig. 6 for various levels of the $\mathrm{I}_{\mathrm{FED}}$ biasing that defines effective resistance in the feedback path of the preamplifier. The curves were obtained working with the ${ }^{109} \mathrm{Cd}$ source. The counts were normalized for the sake of comparison and the curves resulted from averaging over all pixels. The threshold level equal to $0 \mathrm{~V}$ is the baseline. Presence of counts for the negative thresholds results from various levels of undershooting due to differentiation of the preamplifier responses, whose decay time is a function of the feedback resistance.

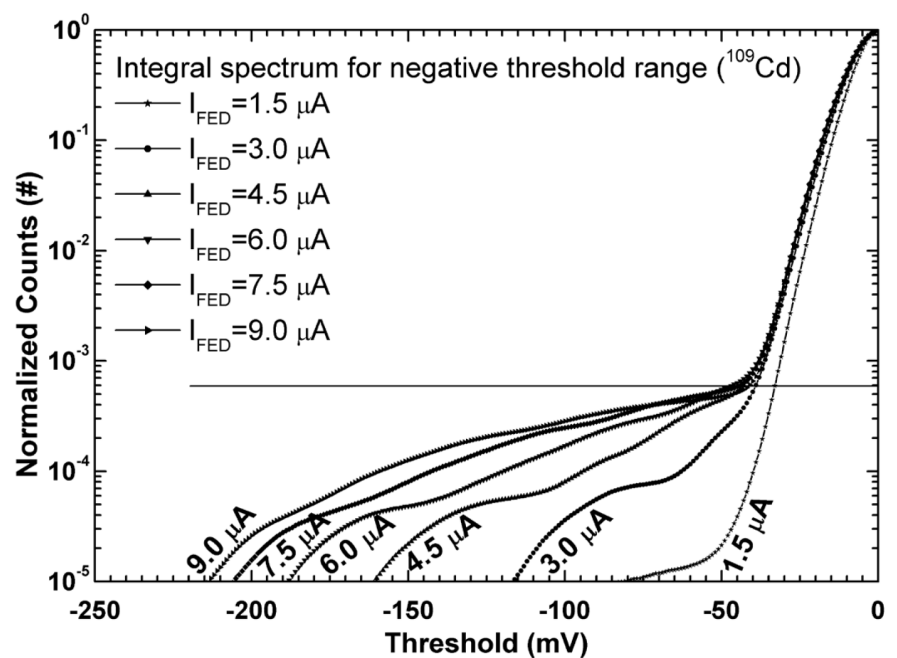

Fig. 6. The part of the integral spectrum, showing signals on the negative side of the threshold scan due to various levels undershooting as a function of bias conditions that define equivalent feedback resistance in the charge sensitive amplifier.

The horizontal line, marked at the level of $5 \times 10^{-4}$ reflects typical cardinalities of counts of events from radioactive sources that are visible in Fig. 7. It is noticeable that using externally defined feedback resistance bias current [5] $\mathrm{I}_{\mathrm{FED}}$ equal to $3.0 \mu \mathrm{A}$ or less results in a significant reduction of undershooting. The $\mathrm{I}_{\mathrm{FED}}$ current equal to $3 \mu \mathrm{A}$ yields the feedback resistance of $50 \mathrm{M} \Omega$ according to the simulations. Adjustments of the feedback resistance in the preamplifier 
influence also the noise performance of the system. The results of measurements of output noise as a function of the external currents used for tuning the preamplifier feedback resistance are shown in Tab. 1. They are provided for channels with and without connections to the sensors diodes and were obtained for the mode, in which the replica CSA is off [5]. The output referred noise values are obtained from a Gaussian fit to the noise data according to the Rice theorem [10].

TABLE I

OUTPUT NOISE AS A FUNCTION OF THE EXTERNAL BIAS CURRENT USED FOR TUNING THE CSA FEEDBACK RESISTANCE (CSA REPLICA OFF) FOR CHANNELS WITH AND WITHOUT CONNECTIONS TO THE SENSOR DIODES

\begin{tabular}{lllllll}
\hline \hline & $\begin{array}{c}\text { noise } \\
(\mathrm{mV})\end{array}$ & $\begin{array}{c}\text { noise } \\
(\mathrm{mV})\end{array}$ & $\begin{array}{c}\text { noise } \\
(\mathrm{mV})\end{array}$ & $\begin{array}{c}\text { noise } \\
(\mathrm{mV})\end{array}$ & $\begin{array}{c}\text { noise } \\
(\mathrm{mV})\end{array}$ & $\begin{array}{c}\text { noise } \\
(\mathrm{mV})\end{array}$ \\
PADS & $1.5 \mu \mathrm{A}$ & $3.0 \mu \mathrm{A}$ & $4.5 \mu \mathrm{A}$ & $6.0 \mu \mathrm{A}$ & $7.5 \mu \mathrm{A}$ & $9.0 \mu \mathrm{A}$ \\
\hline YES & 2.75 & 3.05 & 3.20 & 3.25 & 3.30 & 3.35 \\
NO & 2.01 & 2.20 & 2.27 & 2.32 & 2.40 & 2.45 \\
\hline \hline
\end{tabular}

Based on the data from Fig. 6 and Tab. 1, it was decided to use $\mathrm{I}_{\mathrm{FED}}=3 \mu \mathrm{A}$ for the further tests, targeting optimization of undershooting, noise performance and also leaving enough headroom for the detector leakage current.

\section{Gain and noise}

Testing with X-rays allows for unbiased measurements of genuine parameters, like gain, interpreted as the peak amplitude of a pulse per an unit of input charge, and noise, referred to the input and expressed in charge units. In order to estimate these parameters, scans of thresholds with the fine resolution of $0.5 \mathrm{mV}$ per step were used. The tested devices were exposed to radioactive sources or appropriately adjusted test charges were injected across small, according to the design extraction equal to $1.7 \mathrm{fF}$, capacitors to the input node of the preamplifiers while the sweeping through the threshold levels was executed. Large statistics of counts were registered for every threshold step by acquiring several thousands, typically 5000 or more, of frames per each threshold step. Always a single injection per frame was used to avoid dealing with any parasitic effects. Selected results of the described procedure are plotted in Fig. 7. The curves are shown for a typical single pixel from the tested device with flat field exposure using the ${ }^{109} \mathrm{Cd}$ and ${ }^{55} \mathrm{Fe}$ sources and also injecting test charges by a voltage step equal to $150 \mathrm{mV}$ across the test capacitor. In order to extract peaks from the integral spectra, allowing estimating of the pixels gains, the following procedure was used: a first derivate was calculated numerically on the integral spectrum first, and then, a nested procedure of applying smoothing of the first derivative (simple averaging of two neighboring values), subtracting of a mean value, calculating a second derivative and counting transitions through zero from negative values to positive values of the second derivative was applied. The procedure was concluded when only one transition through zero was left and the transition point was taken as the searched spectral peak. In such a way, no accidental peaks were misinterpreted as spectral peaks and the procedure was stable even on the low statistics data. An example of the first derivative, calculated in the first iteration, and the second derivative, obtained after the last smoothing step, are shown in Fig. 7. A transition through zero of the second derivate is located at the $22.1 \mathrm{keV}$ peak.

The gain is calculated knowing a number of $\mathrm{e}^{-} / \mathrm{h}^{+}$pairs produced in silicon by photons from the sources. The histograms of amplitudes of signals that correspond to $5.9 \mathrm{keV}$, photons, measured for slightly varied biases of the VIPIC1 chip, are shown in Fig. 8. The gain is estimated to $43.12 \mu \mathrm{V} / \mathrm{e}^{-}$with the pixel to pixel dispersions of $5.25 \mu \mathrm{V} / \mathrm{e}^{-}$ for the nominal bias conditions and $51.22 \mu \mathrm{V} / \mathrm{e}^{-}$with the pixel-to-pixel dispersions of $7.48 \mu \mathrm{V} / \mathrm{e}^{-}$for the increased bias in the shaper. It can be noticed that the gain increases with the bias of the shaper increased above the nominal conditions; however pixel-to-pixel dispersions are also larger in this case. A similar tendency, i.e. an increase of gain by about $4 \%$ is observed for increasing of the current conducted by the first transistor. To operate at the higher gain was decided for further steps in order to discriminate signal more easily without trimming of individual threshold levels [6] that was not possible due to wrong shifting of the control bits on some chips. The pixel-to-pixel dispersions of the baseline levels were observed not depending on the bias conditions. The total number of pixels that provide measurable signals could be estimated to about $97 \%$ of all pixels that were bump-bonded to the sensor by applying an arbitrary criterion, i.e. a pixel was considered active if its gain was at least $50 \%$ of the average gain.

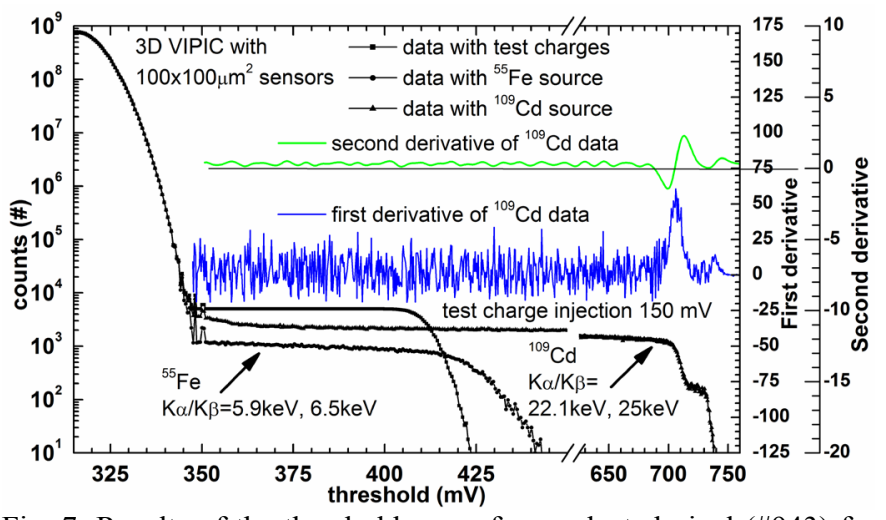

Fig. 7. Results of the threshold scans for a selected pixel (\#943) for injection of test charges, exposures using ${ }^{109} \mathrm{Cd},{ }^{55} \mathrm{Fe}$ sources and the first derivative and the second derivative after smoothing as used for finding spectral peaks.

The ENC values could be obtained by dividing the sigma parameters of the Gaussian fits to the noise part of the integral spectra by the individually estimated gains directly for all pixels connected to the sensor diodes. However, pixels that were not connected to the diodes needed their gains estimated in another way and this could not be done through the measurements involving X-ray photons. Injections of test charges were used instead. First, the test pulses were related to the number of $\mathrm{e}^{-} / \mathrm{h}^{+}$as they would be created if the injected pulses were X-ray photons by comparing and averaging the registered amplitudes of the test pulses with signals from actual X-ray photons on the pixels bump-bonded to the sensor. The mean amplitudes were $82.95 \mathrm{mV}$ and $77.24 \mathrm{mV}$ with pixel to pixel variations equal to $16.57 \mathrm{mV}$ and $9.59 \mathrm{mV}$ for the pixels connected to the sensor diodes in the case of signals originating in actual conversions of photons from the ${ }^{55} \mathrm{Fe}$ source and due to injections of the test pulses, respectively. 
This allowed to calculate an equivalent energy of the test pulses if they were X-ray photons by $77.24 \mathrm{mV} / 82.95 \mathrm{mV} \times$ $5.9 \mathrm{keV}=5.49 \mathrm{keV}$. Following this step, the $5.49 \mathrm{keV}$ equivalent energy was used for extracting of gains and then of noise values for all the pixels in the matrix that were not connected to the sensor diodes.
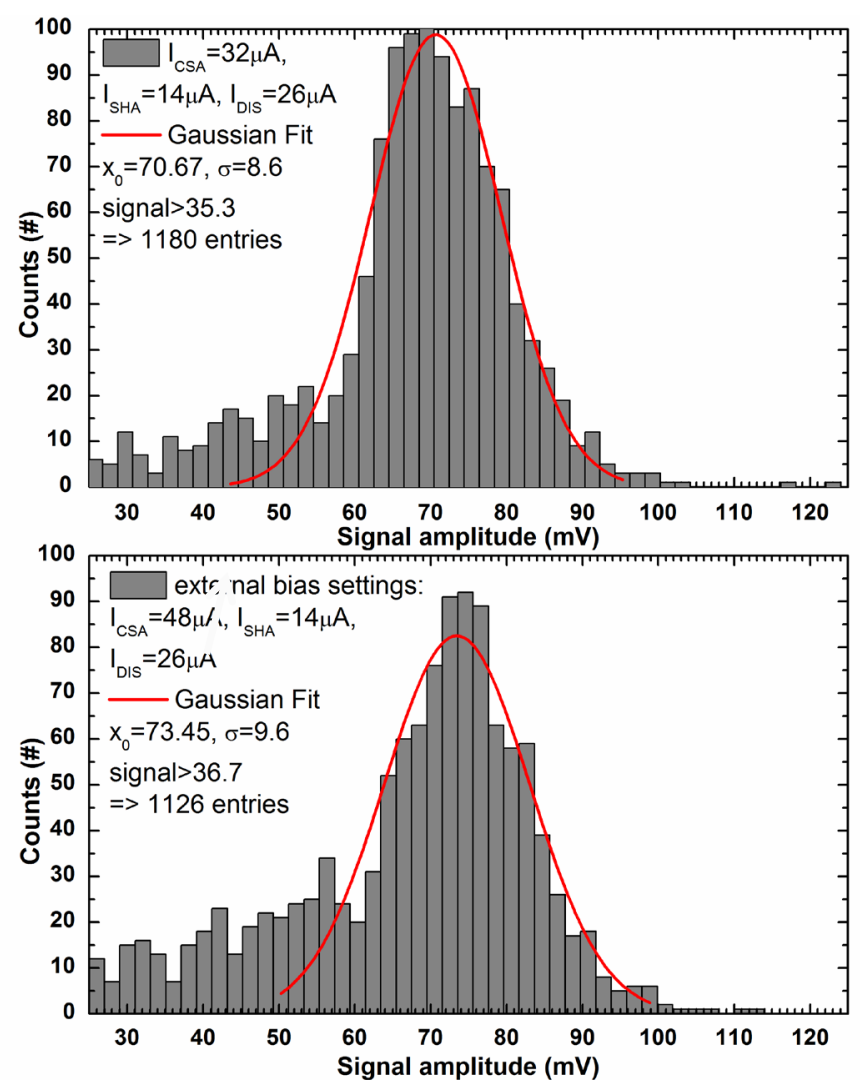

Fig. 8. Histograms of amplitudes of signals that correspond to $5.9 \mathrm{keV}$ photons measured for slightly varied biases of the VIPIC chip.
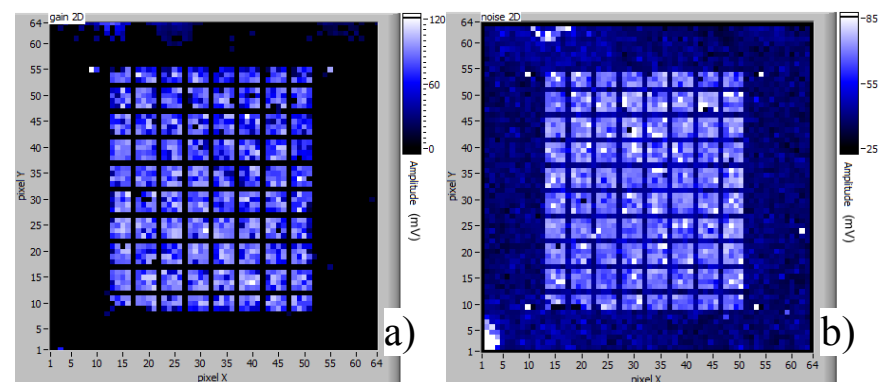

Fig. 9. 2D distribution of a) signal amplitudes corresponding to $5.9 \mathrm{keV} \mathrm{X}$-ray photons and b) equivalent noise charge (ENC) values.

The histograms of the ENC values, extracted for the channels bump-bonded to the sensor diodes and for the pixel channels with the floating inputs, are shown in Fig. 10. The distributions have symmetrical forms that are closely approximating Gaussian functions for both sets of pixels. The mean noise value is $69.6 \mathrm{e}^{-} \mathrm{rms}$ for the channels bump bonded to the sensor diodes and $39.0 \mathrm{e}^{-} \mathrm{rms}$ for the pixels with the floating inputs.

The gain values were also estimated from conversions of $\mathrm{X}$ ray photons from the ${ }^{109} \mathrm{Cd}$ source. The histograms of amplitudes of signals that correspond to $22.1 \mathrm{keV}$ photons, measured for two varied biases of the VIPIC1 chip, i.e. with slightly increased bias in the shaper and for the nominal
The 2D distributions of signal amplitudes, corresponding to $5.9 \mathrm{keV}$ X-ray photons, and ENC values are shown in Fig. 9a and Fig.9b, respectively. It can be noticed that there is no spatial dependence of the gains and the ENC values. The ENC values are about twice larger for the pixel channels bumpbonded to the sensor than for the floating inputs channels.
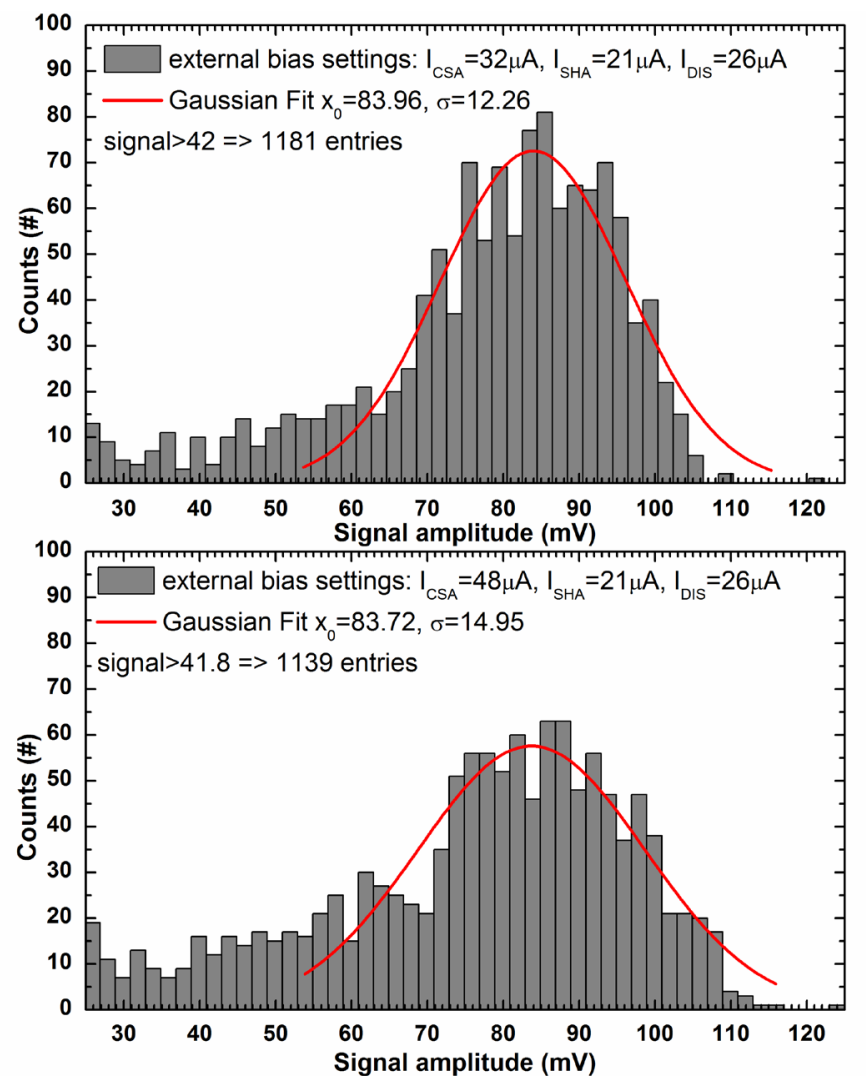

conditions, are shown in Fig. 11a and Fig. 11b, respectively. It can be noticed that the gain values exhibit about a $10 \%$ compression with respect to the estimations made earlier. Such a result was expected as the design allowed worsening of the response linearity above about $18 \mathrm{keV}$.

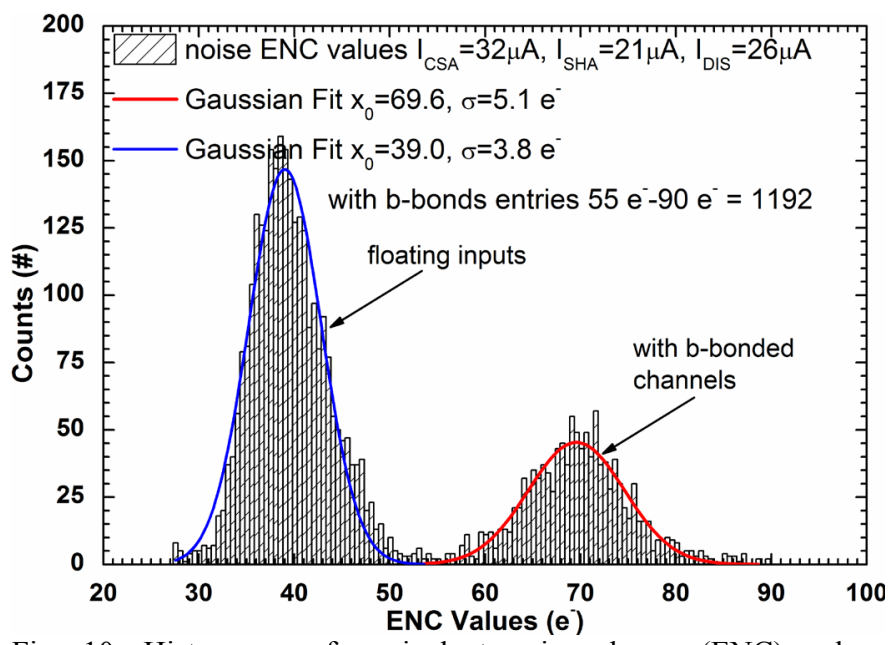

Fig. 10. Histograms of equivalent noise charge (ENC) values extracted for the pixel channels with floating inputs and for the channels bump-bonded to the sensor diodes. 
It can also be noticed that the gain dispersions are larger. Both effects result from reaching saturation, e.g. current starving due to the signal in the source follower in the preamplifier, that occurs nonuniformly in the processing chain.
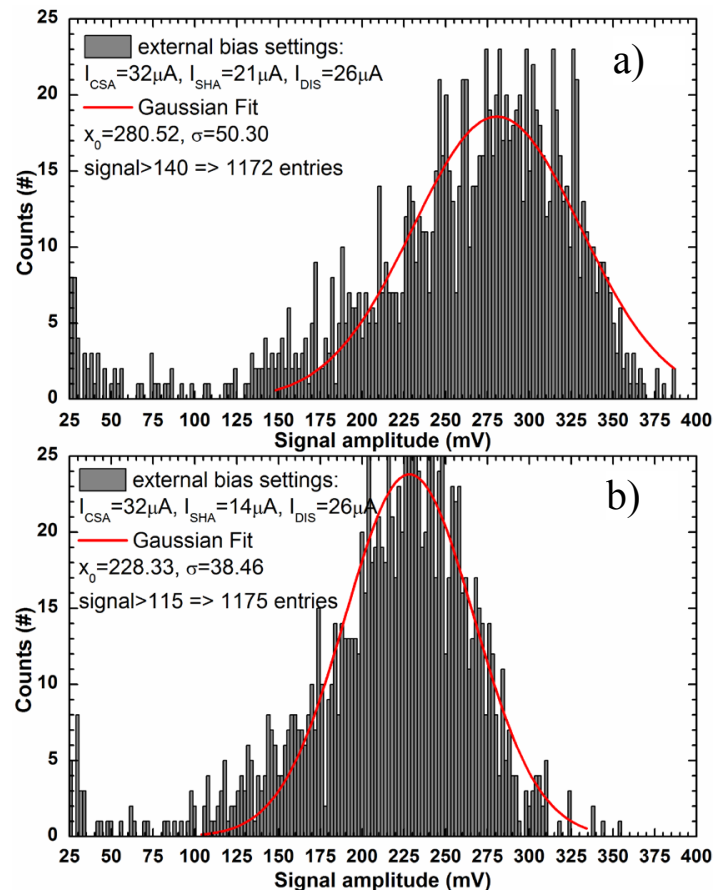

Fig. 11. a) and b) histograms of amplitudes of signals that correspond to $22.1 \mathrm{keV}$ photons measured for 2 slightly varied biases of the VIPIC chip: $\mathrm{I}_{\mathrm{CSA}}=32 \mu \mathrm{A}, \mathrm{I}_{\mathrm{SHA}}=21 \mu \mathrm{A}, \mathrm{I}_{\mathrm{DIS}}=26 \mu \mathrm{A}$ and $\mathrm{I}_{\mathrm{CSA}}=32 \mu \mathrm{A}$, $\mathrm{I}_{\mathrm{SHA}}=14 \mu \mathrm{A}, \mathrm{I}_{\mathrm{DIS}}=26 \mu \mathrm{A}$, respectively.

The 2D distribution of signal amplitudes corresponding to $22.1 \mathrm{keV}$ photons for the first set of the bias parameters is shown in Fig. 12. As it was concluded earlier, no spatial dependencies of the gain values can be observed.

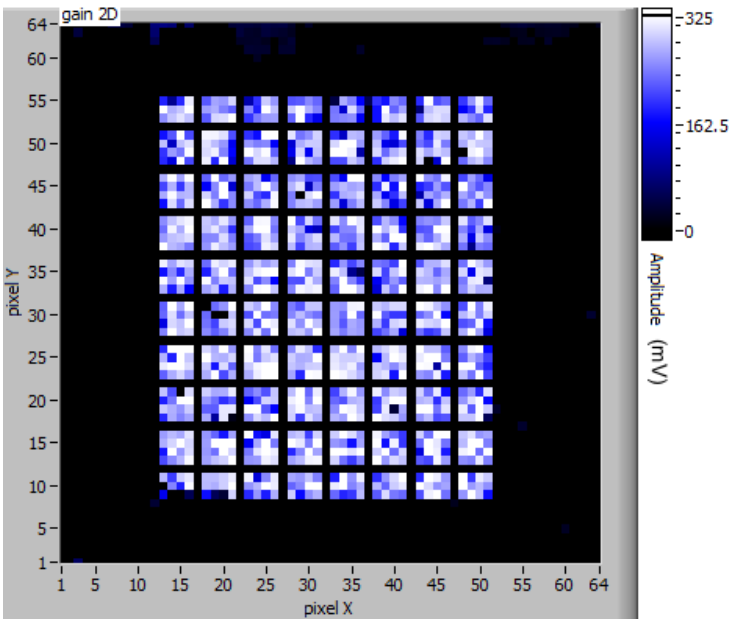

Fig. 12. 2D distribution of signal amplitudes corresponding to 22.1 $\mathrm{keV}$ photons for the set of bias parameters: $\mathrm{I}_{\mathrm{CSA}}=32 \mu \mathrm{A}, \mathrm{I}_{\mathrm{SHA}}=21 \mu \mathrm{A}$ and $\mathrm{I}_{\mathrm{DIS}}=26 \mu \mathrm{A}$.

\section{Discrimination of signals}

The tests of the signal discrimination capabilities above a set threshold level were carried out considering demonstration of the VIPIC1 devices for taking data in the actual experiment on the X-ray beam at a synchrotron radiation facility. Due to the not fully functioning configuration shift register, individual trimming of threshold levels was not possible on most of the chips at the nominal power supply conditions. Thus, it was decided to study how a threshold level could be set and what energy of X-ray photons could be used in order to operate the device without trimmed threshold on the X-ray beam. The baseline dispersions were measured to about $370 \mathrm{e}^{-}$ rms and the gain dispersions amounted to about $240 \mathrm{e}^{-} \mathrm{rms}$. Two flat field images obtained in exposures with the ${ }^{55} \mathrm{Fe}$ source are shown in Fig. 13. The first image, shown in Fig. 13a, was obtained for the threshold setting equal to 12 times the average noise after application of the in-software correction of the threshold offsets. The second image, shown in Fig. 13b, was obtained for the global threshold setting equal to $47 \mathrm{mV}$, above the average baseline.
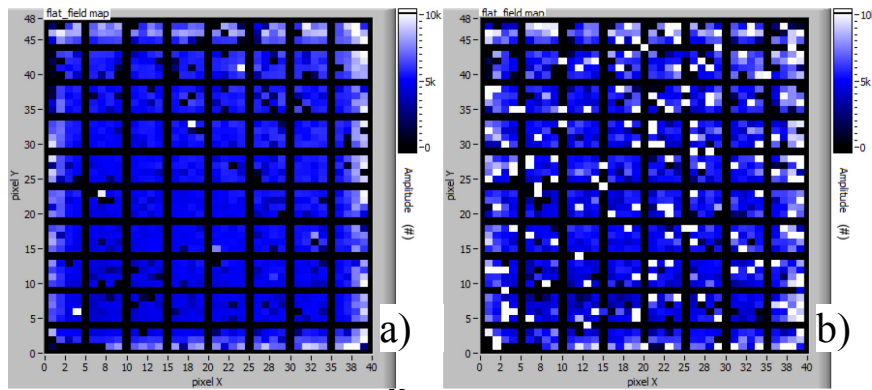

Fig. 13. Flat field image with ${ }^{55} \mathrm{Fe}$ source for a) threshold equal to 12 times the average noise after in-software correction of threshold offsets and b) threshold equal to $47 \mathrm{mV}$ above the average baseline.

The $47 \mathrm{mV}$ setting corresponded to the best estimation of the separation point between the noise and the photon signals. It can be seen that the two images differ significantly as many noise hits were registered. Analogous tests were performed using the ${ }^{109} \mathrm{Cd}$ source. The results are shown in Fig. 14a and in Fig. 14b for the threshold setting equal to 12 times the average noise after application of the in-software correction of the threshold offsets and for the global threshold setting equal to $90 \mathrm{mV}$, above the average baseline, respectively. It can be seen that the two images are not different as no noise hits were registered. The source was purposely located asymmetrically over the VIPIC1 detector in order to expose the illumination gradient.
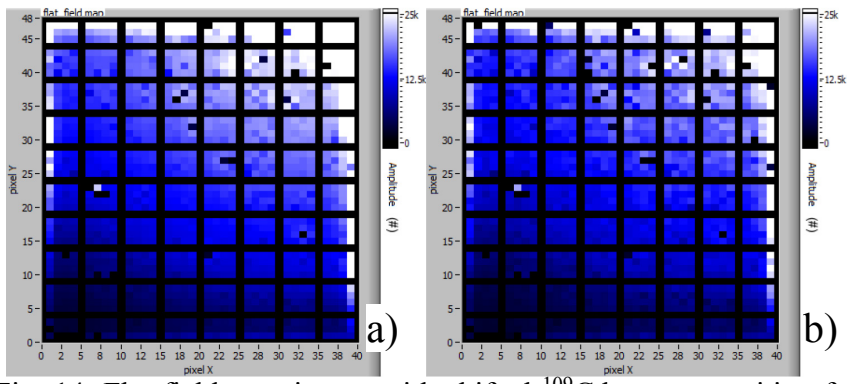

Fig. 14. Flat field type image with shifted ${ }^{109} \mathrm{Cd}$ source position for a) threshold equal to 12 times the average noise after in-software correction of threshold offsets and b) threshold equal to $90 \mathrm{mV}$ above the average baseline.

Based on the performed flat field studies it could be concluded that despite of a lack of full control over the threshold trimming in the VIPIC1 chip, the tests using monochromatic $\mathrm{X}$-ray beam from a synchrotron source can be carried out, 
when the energy is higher than $8 \mathrm{keV}$ (3.5 times baseline dispersions). Also, the pixels with the largest spreads of their baselines can be masked out in order to perform the tests.

\section{CONCLusions}

The results of tests of the VIPIC1 chip bonded to the silicon pixelated sensors were shown and basic parameters of the processing chain, like number of dead channels, gain and noise were quantified. The solder bump bonding procedure that was successfully used in this work is particularly interesting for small scale prototyping, thanks to its compatibility with even tiny dies and not requiring working with wafers as it is often the case. The presented results are the first to show that a three-dimensionally integrated readout for detection of X-rays could be built and its operation and parameters are at least the same as standard 2D counterparts. On the other hand the 3D technology supersedes older approaches by the density of electronics that can be put in a small footprint of a pixel, and by completely new approaches that can be applied in construction of detector systems [1][6]. It should not be lost from sight that VIPIC1 is the first ever prototype of its kind. Thus, some imperfections, like gain dispersions or not fully functioning configuration shift register are present. On the other hand, the gain dispersions are not of the primary importance for a binary system. The device has been sufficiently tested, and under a typical ASIC design cycle, a new submission should be targeted to provide larger size device with corrections of known bugs.

As far as continuation of the efforts related to the VIPIC1 chips, bonding to the full $64 \times 64,80 \mu \mathrm{m}$ pitch pixel sensors is currently underway using the $\mathrm{Ni} \mathrm{DBI}^{\mathbb{R}}$ process. These ultimate 3D devices are also scheduled for testing.

\section{ACKNOWLEDGMENT}

The authors acknowledge Dr. Robert Patti and Dr. Sangki Hong from Tezzaron Semiconductor and Dr. Paul Enquist and Dr. Gill Fontaine from Ziptronix for enabling the 3D-IC technology that was explored to build the VIPIC1 chip, numerous technical discussions and other information provided. Thanks go to Nina Moibenko from Fermilab and Krzysztof Kasiński from AGH-UST for board design, board stuffing, etc. Dedication of Albert Dyer, without whose technical skills, the results would not have been possible, deserves particular acknowledgment.

\section{REFERENCES}

[1] G.Deptuch, M.Demarteau, J.Hoff, R.Lipton, A.Shenai, M.Trimpl, et al., "Vertically Integrated Circuits at Fermilab", IEEE Trans. Nucl. Sci., vol. 57, no. 4, pp. 2178-2186, 2010

[2] G.Grubel, A.Madsen, A.Robert, "X-Ray Photon Correlation Spectroscopy (XPCS)" in R.Borsali, R.Pecora, (Eds.), "Soft Matter Characterization", Springer Netherlands, pp. 953-995, 2008

[3] R.S.Patti, "Three-Dimensional Integrated Circuits and the Future of System-on-Chip Designs", Proceedings of the IEEE, Vol. 94, Issue 6, pp. 1214-1224, June 2006

[4] Q.-Y.Tong, G.Fountain, P.Enquist, "Room Temperature $\mathrm{SiO} 2 / \mathrm{SiO} 2$ Covalent Bonding", Appl. Phys. Lett. 89, 042110, 2006

[5] G.Deptuch, M.Trimpl, R.Yarema, D.P.Siddons, G.Carini, P.Gryboś, et al., "VIPIC IC - Design and Test Aspects of the 3D Pixel Chip", Proceedings of Nuclear Science Symposium, Knoxville, TN, USA, pp. 1540-1543, Oct. 2010
[6] G.Deptuch, G.Carini, P.Gryboś, P.Kmon, P.Maj, M.Trimpl, et al., "Design and Tests of the Vertically Integrated Photon Imaging Chip", IEEE Trans. Nucl. Sci., vol. 61, no. 1, pp. 663-674, 2014

[7] P.Maj, G.Carini, G.Deptuch, P.Gryboś P.Kmon, D.P.Siddons, et al., "Tests of the First Three-Dimensionally Integrated Chip for Photon Science", PoS(Vertex 2012)027

[8] Sangki Hong (Tezzaron), private communication

[9] Paul Enquist (Ziptronix), private communication

[10] S.O. Rice, "Mathematical Analysis of Random Noise", Bell System Technical Journal (Bell Labs) 24 (1): p46-156, 1945 